\title{
Bell nonlocality, signal locality and unpredictability (or What Bohr could have told Einstein at Solvay had he known about Bell experiments)
}

\author{
Eric G. Cavalcanti ${ }^{1,2}$ and Howard M. Wiseman ${ }^{2}$ \\ ${ }^{1}$ School of Physics, The University of Sydney, Sydney NSW 2006, Australia \\ ${ }^{2}$ Centre for Quantum Dynamics, Griffith University, Brisbane QLD 4111, Australia
}

\begin{abstract}
The 1964 theorem of John Bell shows that no model that reproduces the predictions of quantum mechanics can simultaneously satisfy the assumptions of locality and determinism. On the other hand, the assumptions of signal locality plus predictability are also sufficient to derive Bell inequalities. This simple theorem, previously noted but published only relatively recently by Masanes, Acin and Gisin, has fundamental implications not entirely appreciated. Firstly, nothing can be concluded about the ontological assumptions of locality or determinism independently of each other - it is possible to reproduce quantum mechanics with deterministic models that violate locality as well as indeterministic models that satisfy locality. On the other hand, the operational assumption of signal locality is an empirically testable (and well-tested) consequence of relativity. Thus Bell inequality violations imply that we can trust that some events are fundamentally unpredictable, even if we cannot trust that they are indeterministic. This result grounds the quantum-mechanical prohibition of arbitrarily accurate predictions on the assumption of no superluminal signalling, regardless of any postulates of quantum mechanics. It also sheds a new light on an early stage of the historical debate between Einstein and Bohr.
\end{abstract}

\section{Introduction}

Bell's seminal 1964 paper [3] shows that quantum correlations violate the conjunction of locality 1 and determinism. However, there are quantum models that violate locality but maintain determinism (Bohmian mechanics [8] is an example), and models that maintain locality but violate determinism (standard operational quantum theory is an example). Thus nothing can be concluded from Bell's theorem about locality or determinism independently of each other.

Here we show that a remarkable conclusion can be reached by deriving Bell inequalities from a different set of assumptions: signal locality (i.e. the impossibility to send signals faster than light) and predictability (i.e. the assumption that one can predict the outcomes of all possible

\footnotetext{
${ }^{1}$ Our usage of 'locality' here is the same as that of Bell in 1964: "that the result of a measurement on one system be unaffected by operations on a distant system". This assumption is sometimes referred to as "parameter independence" and is strictly weaker than the assumption of "local causality", which was later introduced by Bell [5] to show that determinism does not need to be assumed, and that no locally causal model (deterministic or otherwise) can reproduce quantum mechanics. See also Sec. 4 .
} 
measurements to be performed on a system). These assumptions are purely operational, that is, they refer to operational quantities only, in contrast to the ontological assumptions of locality and determinism, which refer to properties of a hidden-variable model that reproduces the observations. In particular, signal locality is an uncontroversial consequence of relativity, as opposed to locality, which can be violated by the underlying hidden-variable model while still maintaining signal locality at the observable level (as is the case with Bohmian mechanics, which reproduces quantum mechanics).

This derivation therefore allows us to confidently conclude that predictability must fail for experiments that violate Bell inequalities. In other words, it allows the conclusion that, if it is impossible to signal faster than light, then it is impossible to predict the outcomes of experiments that violate Bell inequalities, even if those outcomes might be determined by an underlying hidden-variable model.

This work builds on and clarifies some recent results. In the 1994 paper where they suggested Bell nonlocality as a "natural" axiom for quantum theory and brought to light the existence of stronger-than-quantum correlations compatible with signal locality, Popescu and Rohrlich [16] commented on an unpublished result of Aharonov to the effect that "relativistic causality" and "nonlocality" imply "indeterminism". Here we would like to point out that, strictly speaking, this claim is incorrect: the conjunction of signal locality (i.e., what they meant by "relativistic causality" and Bell-inequality violation (what they meant by "nonlocality") implies the failure of predictability, but not of determinism. While Aharonov's result was presumably correct with the appropriate translation, the choice of words seems to imply they did not make at that stage the distinction between determinism and predictability, and this work should clarify the importance of doing so.

This result also relates to the mechanism underlying the security of quantum key distribution based on signal locality and Bell inequality violations, as proven by Barrett, Hardy and Kent [2]. In the discussion of their result, they cited a paper of Valentini [21] that purported to show that "any state that is deterministic and nonlocal allows signalling". Since what they meant by "determinism" was the same as we here mean by "predictability", this is essentially the contrapositive of the present result. However, this was strictly speaking not proven in Valentini's paper. What he claimed to have proven was that for all nonlocal deterministic hidden-variable theories, a violation of signal locality occurs if and only if the theory allows a distribution of hidden variables different from that which is needed to reproduce quantum mechanics, i.e., if and only if the distribution is different from that of "quantum equilibrium" 2 .

To our knowledge, the first correct published proof of this result can be found in an article by Masanes, Acin and Gisin, that, following the suggestion of Popescu and Rohrlich, studied general properties of non-signalling theories [14]. However, they also did not distinguish between predictability and determinism (like Aharonov and Valentini, they used "determinism" to refer to what we call "predictability"). The present discussion should thus serve to clarify the conceptual basis and importance of this result 3 .

\footnotetext{
${ }^{2}$ Besides, Valentini's main result seems to be flawed. He seems to have only proven the weaker result that there exist distributions over the hidden variables which would allow signalling, as the reader may be convinced by analysing the first equation on page 276 of [21].

${ }^{3}$ After a first version of this work was posted on the arXiv (arXiv:0911.2504 1 ), another arXiv post (arXiv:0911.3427 1 , eventually published in Nature [15]) underscored the importance of the distinction by proposing a scheme to generate random numbers certified by violation of a Bell inequality. Those results are however somewhat distinct from the present one, in that to derive bounds on the randomness of the output
} 
This result also has an interesting didactical implication for the famous dialogues between Einstein and Bohr on the foundations of quantum theory at the 1927 and 1930 Solvay conferences. At this stage, prior to the 1935 Einstein, Podolsky and Rosen paper [10] and the concept of entanglement that was born from it, it seems that Einstein attempted to attack the validity of quantum mechanics, not only its completeness, by concocting thought experiments aimed at obtaining a violation of the uncertainty principle [9]. However, by carefully applying the uncertainty principle to the experimental apparatuses as well as the systems being measured, Bohr showed that the uncertainty principle was consistent; that is, he showed that if the uncertainty principle is valid for the degrees of freedom of all measuring apparatuses then those measuring apparatuses can't be used to violate the uncertainty principle associated to a quantum system. Interestingly, in the last of such attempts from Einstein, Bohr used Einstein's own theory of general relativity to demonstrate the consistency of quantum mechanics. Could Bohr have gone beyond that, and argued, with appeal to independent fundamental principles, that the uncertainty principle must be valid?

In the remainder of this paper we will answer this question in the affirmative, and show that an uncontroversial consequence of Einstein's theory of special relativity (signal locality) and some raw experimental observations (namely, the violation of Bell inequalities) lead to a weak version of the uncertainty principle - perfect predictability of natural phenomena must be impossible, regardless of any of the postulates of quantum mechanics.

This paper is structured as follows. In Section $2 \mathrm{I}$ introduce the basic concepts and notation required for the main result. The main result is proven in Section 3, followed by a discussion of the result and concluding remarks in Section 4

\section{Experimental metaphysics}

Abner Shimony coined the term "experimental metaphysics" to refer to the field of study pioneered by Bell, where general metaphysica 4 concepts such as "local causality" are shown to lead to experimental constraints which can be tested in the laboratory. In the following we will introduce the concepts required to prove our main result.

The experimental setup considered here involves two spatially separated observers, Alice and Bob, who can perform a number of measurements and observe their outcomes. For each pair of systems they perform measurements upon, the choices of measurement settings and their respective outcomes occur in regions which are space-like separated from each other, so that no signal travelling at a speed less than or equal to that of light could connect any two of them. For each pair of systems, we will denote by $a$ and $b$ Alice's and Bob's respective measurement settings, and by $A$ and $B$ their corresponding observed outcomes. Note that here we are following the notational convention Bell established in 1964 [3]. Each pair of systems is prepared by an agreed-upon reproducible procedure $\kappa$ (which in quantum mechanics would define a quantum state for the pair of systems).

of a Bell experiment, those authors assumed the validity of the laws of quantum mechanics. Here no such assumption is made (but consequently no bound is given on the randomness or unpredictability of the output).

${ }^{4}$ For the physicist trained to be suspicious of philosophical terms, note that in this context the term 'metaphysics' does not refer to mysticism, but to the study of formal and empirical properties of physical theories themselves. (Experimental) metaphysics is to physics as metamathematics is to mathematics. It includes the study of sets of physical theories which fail to represent observations, where this analysis can be illuminating in understanding those that do not. 
We will define a phenomenon, for a given preparation procedure $\kappa$, by the relative frequencies

$$
f(A, B \mid a, b, \kappa) .
$$

for all measurements $a, b$, and corresponding outcomes $A, B$. Note that this definition does not assume a frequentist interpretation of probabilities. It simply acknowledges that in any physical experiment, the actual phenomenon observed is encoded by those relative frequencies (with some associated statistical uncertainty that can in principle be made arbitrarily small). When an equation involving variables appears, it is to be understood that the equality holds for all values of those variables.

We will say that a phenomenon is predictable, or that it satisfies predictability if and only if

$$
f(A, B \mid a, b, \kappa) \in\{0,1\} .
$$

Now of course it is a consequence of the postulates of quantum mechanics that there are unpredictable phenomena, according to Heisenberg's uncertainty principle. However, we will see that this conclusion can be reached independently of the postulates of quantum theory, by using signal locality, i.e. the assumption that

$$
f(A \mid a, b, \kappa)=f(A \mid a, \kappa),
$$

and the corresponding equation for $B$. Note that all the definitions above are purely operational, i.e. they refer to operationally defined, observable quantities only.

What Bell did was to consider, as did Einstein, Podolsky and Rosen, the possibility that there might be further variables (in addition to $a, b$ and $\kappa$ ) that are relevant to the phenomenon observed. We represent any such variables by the symbol $\lambda_{5}^{5}$. They are not fully determined by the preparation procedure $\kappa$, and as such may be deemed "hidden variables". A more appropriate terminology is "ontic variables", since they represent any "real" physical state of the parts of the world which are relevant to the experiments being considered. This terminology also emphasises the distinction between $\kappa$ and $\lambda: \kappa$ represents the relevant variables known by Alice and Bob (or by whichever party is describing the phenomenon), and $\lambda$ represents the variables that are objectively relevant to the experiments considered, regardless of whether they are known or even knowable6.

An ontological model [20, 17, 12, 11] for a phenomenon is one in which the phenomenon can be explained by considering ontic variables. It consists of the set $\Lambda$ of values of $\lambda$, together with a probability density $\mu(\lambda \mid \kappa)$ for every preparation procedure $\kappa$ and a specification of

$$
P(A, B \mid a, b, \kappa, \lambda)
$$

which reproduces the phenomenon by

$$
\int_{\Lambda} d \lambda \mu(\lambda \mid \kappa) P(A, B \mid a, b, \kappa, \lambda)=f(A, B \mid a, b, \kappa) .
$$

\footnotetext{
${ }^{5}$ Note that considering the possibility that further variables exist is not the same as assuming that they exist; there is no "hidden-variable assumption" in Bell's theorem.

${ }^{6}$ Indeed it is a corollary of the present result that in any deterministic model that reproduces quantum theory the ontic variables must be necessarily unknowable.
} 
Note that in Eq. (5) we have used the assumption of free variables, which is the assumption that the choices of experiment $a, b$, can be conditioned on variables which are uncorrelated with $\lambda$. Formally, this is the assumption that

$$
\mu(\lambda \mid a, b, \kappa)=\mu(\lambda \mid \kappa) .
$$

This is sometimes called the "free will" assumption, but that terminology seems to imply something about human capabilities that doesn't seem to be necessary for the purposes at hand.

We are now ready to define properties of models. A model is said to satisfy locality if and only if

$$
P(A \mid a, b, \kappa, \lambda)=P(A \mid a, \kappa, \lambda),
$$

plus the corresponding equation for $B$ ? . A model is said to satisfy signal locality if and only if

$$
P(A \mid a, b, \kappa)=P(A \mid a, \kappa),
$$

plus the corresponding equation for $B$. Note that the left-hand-side of the equation here is defined within the model as the left-hand-side of Eq. (5). We say that locality is an ontological concept because it refers to ontic variables in its definition, while signal locality is an operational concept because it only refers to operational variables in its definition. Note that a model satisfies signal locality if and only if the corresponding phenomenon also does, i.e., if and only if $f(A \mid a, b, \kappa)=f(A \mid a, \kappa)$.

To see that a violation of Eq. (8) would imply the possibility to transmit signals between the experimental sites, note that if the phenomenon violates signal locality, then there exist at least two possible choices of setting $b, b^{\prime}$ such that $f(A \mid a, b, \kappa) \neq f\left(A \mid a, b^{\prime}, \kappa\right)$. Therefore by looking at the frequency of outcomes of $A$ in a large enough ensemble (and in principle it is possible for Alice to make all of the measurements in her ensemble space-like separated from all measurements in Bob's ensemble), Alice can determine with arbitrary accuracy what setting Bob has chosen, thus allowing Bob to send signals to Alice.

Violation of locality, on the other hand, does not imply signalling, since in general only the probabilities for Alice's outcomes conditioned on the hidden variables depend on the choice of experiments at Bob's site. But since those hidden variables can be unknowable in principle, that kind of non-locality cannot necessarily be used to transmit signals. Bohmian mechanics is an example of a model that violates locality but not signal locality.

We now come to the crucial distinction between the concepts of determinism and predictability. A model is said to be deterministic, or to satisfy determinism 8 , if and only if

$$
P(A, B \mid a, b, \kappa, \lambda) \in\{0,1\},
$$

\footnotetext{
${ }^{7}$ We remind the reader that this corresponds to Bell's definition of locality introduced in 1964 [3]. There are instances in less formal publications, for example Ref. [4], where Bell used the term "locality" more loosely to mean the property which quantum mechanics lacked, as revealed by his theorem. However these are very much the exception, and from 1976 on, Bell almost invariably used the term "local causality" for this property.

8 There is another useful sense of determinism which needs to be distinguished from the one we are using here. Quantum mechanics can be said to be deterministic in the sense that for a closed system the state at a later time is determined through unitary evolution by the state at an initial time. However, operational quantum mechanics is not deterministic in the sense used in this paper, since of course a system undergoing a measurement interaction is no longer a closed system.
} 
which implies that $A$ and $B$ can be specified as functions as follows:

$$
A=A(a, b, \kappa, \lambda), B=B(a, b, \kappa, \lambda) .
$$

On the other hand, a model is said to be predictable, or to satisfy predictability if and only if

$$
P(A, B \mid a, b, \kappa) \in\{0,1\} .
$$

This implies that $A$ and $B$ can be specified as functions as follows:

$$
A=A(a, b, \kappa), B=B(a, b, \kappa) .
$$

Determinism, like locality, is an ontological concept while predictability, like signal locality, is an operational concept. As with signal locality, a model is predictable if and only if the phenomenon it reproduces is predictable. It is interesting to note that it is impossible for a phenomenon to violate determinism by itself: every phenomenon can be given a deterministic model, simply by postulating a sufficient number of hidden variables.

Obviously, predictability implies determinism, but the converse is not true. Heisenberg's uncertainty principle implies that no model that reproduces quantum mechanics is predictable, but it imposes no limitation on determinism. In fact, there are models of quantum mechanics (Bohmian mechanics is an example) which are deterministic, but the following theorem shows that they must nevertheless be unpredictable (or violate signal locality).

\section{Bell inequalities from signal locality and predictability}

John Bell's 1964 theorem [3] demonstrated that the conjunction of the concepts of locality and determinism as defined above leads to a set of experimental constraints known as Bell inequalities, and that some predictions of quantum mechanics regarding entangled states violate those inequalities. For present purposes we just need to point out that to derive a Bell inequality it is sufficient to require that the joint probabilities of experimental outcomes given by a model is factorisable, i.e., that it can be written in the form

$$
P(A, B \mid a, b, \kappa, \lambda)=P(A \mid a, \kappa, \lambda) P(B \mid b, \kappa, \lambda) .
$$

This equation is one way of expressing the condition on which Bell bestowed the name local causality [5].

To see that locality and determinism imply factorisability, note that the joint probabilities of any model can be written as $P(A, B \mid a, b, \kappa, \lambda)=P(A \mid B, a, b, \kappa, \lambda) P(B \mid a, b, \kappa, \lambda)$. If the model is deterministic, then $P(A \mid B, a, b, \kappa, \lambda)=P(A \mid a, b, \kappa, \lambda)$, because $A$ is already determined by $a, b, \kappa, \lambda$. From the definition of locality given by Eq. (7) we thus arrive at the factorisable model of Eq. (13).

We now arrive at our main result, that the conjunction of signal locality and predictability also allow one to derive Bell inequalities. The proof is simple. The definition of predictability (Eq. (11)), implies that

$$
P(A, B \mid a, b, \kappa, \lambda)=P(A, B \mid a, b, \kappa)=P(A \mid B, a, b, \kappa) P(B \mid a, b, \kappa) .
$$

The first equality follows because the second expression must be either 0 or 1 , thus those probabilities cannot be altered by conditioning on $\lambda$. The second equality follows from the 
definition of conditional probabilities. Eq. (11) also implies that $P(A \mid B, a, b, \kappa)=P(A \mid a, b, \kappa)$, since $A$ is already specified by $a, b, \kappa$. Thus $P(A, B \mid a, b, \kappa, \lambda)=P(A \mid a, b, \kappa) P(B \mid a, b, \kappa)$. Assuming signal locality, i.e. Eq. (요), we obtain

$$
P(A, B \mid a, b, \kappa, \lambda)=P(A \mid a, \kappa) P(B \mid b, \kappa) .
$$

This has the factorisable form of (13), as we set out to prove. To make that more explicit, note that this is equivalent to a model with $P(A \mid a, \kappa, \lambda)=P(A \mid a, \kappa)$ and $P(B \mid b, \kappa, \lambda)=P(B \mid b, \kappa)$ for all $\lambda$. Therefore, signal locality plus predictability imply factorisability, and thus Bell inequalities.

\section{Discussion and conclusion}

Bell was adamant in stressing that his concept of locality was distinct from the concept of signal locality. In fact, he rejected the importance of the concept of signal locality, as he understood that it was hard to talk about it without using apparently anthropocentric terms like 'information' and 'controllability':

"Suppose we are finally obliged to accept the existence of these correlations at long range [...]. Can we then signal faster than light? To answer this we need at least a schematic theory of what we can do, a fragment of a theory of human beings. Suppose we can control variables like $a$ and $b$ above, but not those like $A$ and $B$. I do not quite know what 'like' means here, but suppose the beables somehow fall into two classes, 'controllables' and 'uncontrollables'. The latter are no use for sending signals, but can be used for reception." [5].

And he rejects the idea that signal locality could be taken as the fundamental limitation imposed by relativity:

"Do we have to fall back on 'no signalling faster than light' as the expression of the fundamental causal structure of contemporary theoretical physics? That is hard for me to accept. For one thing we have lost the idea that correlations can be explained, or at least this idea awaits reformulation. More importantly, the 'no signalling...' notion rests on concepts which are desperately vague, or vaguely applicable. The assertion that 'we cannot signal faster than light' immediately provokes the question:

Who do we think we are?

We who can make 'measurements', we who can manipulate 'external fields', we who can signal at all, even if not faster than light? Do we include chemists, or only physicists, plants, or only animals, pocket calculators, or only mainframe computers?" [6].

Bell is right in that we cannot define signal locality without referring to operational or epistemic concepts, but we find this situation more acceptable than Bell did. The reason is that 'information' and thus 'signal' are themselves operational concepts. We do indeed need to know which variables are controllable, and which variables are knowable, in order to define 
signal locality. However, while "controllable" and "knowable" may be as philosophically problematic as "information", they are also just as pragmatically clear and useful. Besides, the use of these terms does not need to imply an anthropocentric view of physics as he seemed to be worried in the passage above. There seems to be no fundamental difficulty in talking about, say, machines "knowing" or "controlling" the variables defined in the previous section.

Since experiments routinely violate Bell inequalities (up to some open loopholes), we can conclude, through the theorem proven in the previous section, that signal locality and predictability cannot both the true. Now this allows an interesting conclusion. Bell showed in 1964 that locality and determinism cannot both be true. Some people have taken that to imply indeterminism, choosing to keep locality. However, a deterministic model (Bohmian mechanics) exists that reproduces quantum theory, while violating locality. One may reject this or similar models on other grounds (e.g. elegance, symmetry, etc.), but Bell's theorem cannot be used to that end. Furthermore, Bell later showed that the stronger concept of local causality also implies Bell inequalities and must therefore be false. So even if one chooses to reject determinism, the resulting indeterminism still has a nonlocal character, by Bell's later argument. The result is thus that nothing can be concluded separately about the ontological claims of determinism and locality from the violation of Bell inequalities.

However, the validity of signal locality has a much less controversial footing. Even those who believe in the violation of locality would generally agree that the operational assumption of signal locality must be valid, and that while a violation of locality may have a "peaceful coexistence" [19] with relativity, a violation of signal locality would be in direct contradiction with it. Therefore the full weight of the violation of Bell inequalities can be confidently transferred to the violation of the operational concept of predictability: there can be no predictable model that allows violation of a Bell inequality. To the extent that those violations actually occur in nature, we can conclude that the world is indeed fundamentally unpredictable.

Furthermore, we arrive at this conclusion without needing to assume anything about quantum mechanics. It is simply a consequence of bare experimental data and an uncontroversial consequence of the theory of relativity. Bohr, of course, could not have known about this result before Bell, but if he did, he could have much more easily convinced Einstein that his attempts to make quantum phenomena predictable were bound to fai 9 . And in another twist of irony, he could have again used Einstein's own theory of relativity to prove him wrong.

\section{Acknowledgments}

We are grateful to Rob Spekkens for useful feedback and encouragement. This work was partly supported by the Australian Research Council Discovery grant DP0984863 and Discovery Early-Career Researcher Award DE120100559.

\footnotetext{
${ }^{9}$ Although, of course, this would have no implication for Einstein's later attacks on the completeness of quantum theory. Of course all that we have said about the Bohr-Einstein debates accepts that Bohr's narrative of the events is accurate. It has been argued by Howard [13] and by Bacciagaluppi and Valentini [1] that Bohr misunderstood Einstein's arguments, and that actually Einstein was arguing for incompleteness (based on nonseparability), rather than incorrectness, the whole time.
} 


\section{References}

[1] G. Bacciagaluppi and A. Valentini. Quantum theory at the crossroads : reconsidering the 1927 Solvay conference. Cambridge University Press, 2009.

[2] J. Barrett, L. Hardy, and A. Kent. No signaling and quantum key distribution. Physical Review Letters, 95(1):010503, 2005.

[3] J. S. Bell. On the Einstein-Podolsky-Rosen paradox. Physics, 1:195, 1964.

[4] J. S. Bell. Einstein-Podolsky-Rosen Experiments. Proceedings of the symposium on Frontier Problems in High Energy Physics, 33-45. Pisa, June 1976. Reproduced in Ref. 7].

[5] J. S. Bell. The Theory of Local Beables Epistemological Lett. 9, 1976. Reproduced in Ref. [7].

[6] J. S. Bell. La nouvelle cuisine in Between Science and Technology, eds. A. Sarlemijn and P. Kroes. Elsevier Science Publishers, 1990. Reproduced in Ref. 7].

[7] J. S. Bell. Speakable and Unspeakable in Quantum Mechanics, 2nd Ed. Cambridge University Press, 2004.

[8] David Bohm. A Suggested Interpretation of the Quantum Theory in Terms of "Hidden" Variables. I. Physical Review, 85(2):166-179, 1952.

[9] N. Bohr. Discussion with Einstein on epistemological problems in atomic physics. in Ref. [18], pp. 201-241; reproduced in [22].

[10] A. Einstein, B. Podolsky, and N. Rosen. Can quantum-mechanical description of physical reality be considered complete? Physical Review, 47:777, 1935.

[11] N. Harrigan and T. Rudolph. Ontological models and the interpretation of contextuality. arXiv:0709.4266, 2007.

[12] N. Harrigan, T. Rudolph and S. Aaronson. Representing probabilistic data via ontological models. arXiv:0709.1149, 2008.

[13] D. Howard. 'Nicht sein kann was nicht sein darf,' or the Prehistory of EPR, 1909-1935: Einstein's Early Worries about the Quantum Mechanics of Composite Systems. In Sixty-Two Years of Uncertainty: Historical, Philosophical, and Physical Inquiries into the Foundations of Quantum Mechanics, Proceedings of the 1989 Conference,"Ettore Majorana" Centre for Scientific Culture, International School of History of Science, Erice, Italy, 5-14 August. Arthur Miller, ed. New York: Plenum, pp. 61-111.

[14] L. Masanes, A. Acín, and N. Gisin. General properties of nonsignaling theories. Physical Review A, 73(1):012112, 2006.

[15] S. Pironio, A. Acín, S. Massar, A. Boyer de la Giroday, D. N. Matsukevich, P. Maunz, S. Olmschenk, D. Hayes, L. Luo, T. A. Manning, and C. Monroe. Random numbers certified by Bell's theorem. Nature, 464:1021-1024, 2010.

[16] S. Popescu and D. Rohrlich. Quantum Nonlocality as an axiom. Foundations of Physics, 24(3):379-385, 1994.

[17] T. Rudolph. Ontological models for quantum mechanics and the Kochen-Specker theorem. arxiv:quant-ph/0608120, 2006.

[18] P. A. Schilpp (ed.). Albert Einstein: Philosopher-Scientist. Library of the Living Philosophers, Evanston, 1949.

[19] A. Shimony. In J. T. Cushing and E. McMullin, (eds.). Philosophical Consequences of Quantum Theory, pp. 25-37. University of Notre Dame Press, Notre Dame, 1989. 
[20] R. W. Spekkens. Contextuality for preparations, transformations, and unsharp measurements. Physical Review A, 71(5):052108, 2005.

[21] A. Valentini. Signal-locality in hidden-variables theories. Physics Letters A, 297:273-278, 2002.

[22] J. A. Wheeler and W. H. Zurek, editors. Quantum Theory and Measurement. Princeton University Press, 1983. 